VPA $(U=576,=-4 \cdot 13, P=0 \cdot 000$, mean rank $35 \cdot 20 v$. $58 \cdot 84$, SA $v$. White, respecively). Weekend activity showed no ethnic difference in MPA or LPA $(U=961,=-0.48$, $P=0.319, \quad U=908, \quad=-0.896, \quad P=0 \cdot 187$, respectively). However, SA children spent less time in VPA at weekends than White $\mathrm{EU}(U=767,=-2 \cdot 05, P=0 \cdot 020$, mean $=39 \cdot 68$ $v$. 50.96, SA $v$. White EU, respectively).
Conclusions: Our results suggest children from ethnic minorities exercise differently. Further work is needed to explore the reasons for these differences and how these may impact on metabolic health.

Funding: Not disclosed.

\title{
38 - Ground reaction forces in overweight children
}

\author{
K Sheehan ${ }^{1}$, EF Roche ${ }^{2,3}$ and J Gormley ${ }^{1}$ \\ 'Department of Physiotherapy, University of Dublin, Trinity College Dublin, Ireland: 'The National Children's Hospital, \\ AMNCH, Tallaght, Dublin, Ireland: ${ }^{3}$ Department of Paediatrics, University of Dublin, Trinity College Dublin, Ireland
}

Aim: To investigate the relationship between expected and recorded vertical (V), anterior-posterior (AP) and medial-lateral (ML) ground reaction forces (GRF) in overweight children.

Participants: Subjects were recruited from the paediatric weight management clinic at the Adelaide and Meath Hospital, Dublin. Subjects aged 7-17 years and with a BMI $>25 \mathrm{~kg} / \mathrm{m}^{2}$ were included in the study. Subjects who presented with a leg length discrepancy $>2$ inches were excluded from the study.

Method: Subjects attended the gait laboratory at the Trinity Centre for Health Sciences. Anthropometry was conducted on arrival. Subjects fitted with surface markers walked between two Coda cameras (Charnwood Dynamics LtD, Rothley, UK) on a $10 \mathrm{~m}$ platform embedded with two AMTI force plates (Advanced Mechanical Technology, Inc., Watertown, MA, USA). Observed maximum GRF were collected manually from graphs. Expected maximum GRF were calculated using percentage body weight values reported by Cottalorda et al. (2003).

Analysis: Paired $t$ tests were used to compare means between observed and expected V, AP and
ML GRF. A $P$-value $<0 \cdot 05$ was considered statistically significant.

Results: Eight males and fifteen females completed the

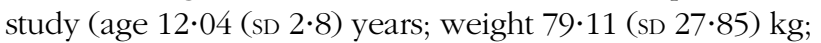
height 157.87 (sD 14.05) cm; BMI $29 \cdot 51(\mathrm{sD} 4 \cdot 55) \mathrm{kg} / \mathrm{m}^{2}$ ). Observed AP and ML GRF were found to be significantly greater than expected GRF, $P=0 \cdot 016$ and $P=0 \cdot 000$, respectively. No significant difference between observed and expected V GRF were noted $P=0 \cdot 615$.

Conclusions: From the present study, overweight children incur greater than anticipated AP and ML GRF than anticipated for their body weight. These forces may predispose this group to musculoskeletal disorders. This information should be noted when prescribing exercise to overweight children.

Funding: Research relating to this abstract was funded by Trinity College Dublin.

Reference: Jerome Cottalorda, Abderrehmane Rahmani, Mountaga Diop, Vincent Gautheron, Eric Ebermeyer and Alain Belli (2003). Influence of school bag carrying on gait kinetics. Journal of Pediatric Orthopaedics B, 12 (6): 357-364.

\section{9 - Barriers to participation in physical education among obese pupils}

\author{
ME Jacobson, CJ Laws and JA Potter \\ University of Chichester, West Sussex, England
}

Introduction: Physical education's (PE) profile has recently been raised in the fight against childhood obesity. The present study determined overweight/obesity prevalence among pupils recruited from five secondary schools in the South of England and investigated their barriers to PE participation.

Methods: Body fat\% of 380 pupils (202 boys and 178 girls) aged 11-15 years, were measured using bioelectric 